\title{
Detection of an Optical Signal Using Difference Frequency Generation in a Periodically Poled $\mathrm{LiTaO}_{3}$ Microwave Waveguide
}

\author{
Hiroshi Murata and Yasuyuki Okamura \\ Graduate School of Engineering Science, Osaka University, 1-3 Machikaneyama, Toyonaka, Osaka 560-8531, Japan
}

Correspondence should be addressed to Hiroshi Murata, murata@ee.es.osaka-u.ac.jp

Received 9 June 2008; Accepted 25 August 2008

Recommended by Chang-qing Xu

\begin{abstract}
The detection of an optical signal modulated at $15 \mathrm{GHz}$ was demonstrated experimentally by using difference frequency generation based on a second-order nonlinear optical effect in a periodically poled $\mathrm{LiTaO}_{3}$ microwave rectangular waveguide. The measured frequency dependence of the generated microwave signal was in good agreement with the theoretically expected result. An interesting application of the proposed device is the detection of high-speed optical clock detection.
\end{abstract}

Copyright (c) 2008 H. Murata and Y. Okamura. This is an open access article distributed under the Creative Commons Attribution License, which permits unrestricted use, distribution, and reproduction in any medium, provided the original work is properly cited.

\section{Introduction}

The detection of a high-speed optical signal is indispensable in many optoelectronic systems. The operational principle of standard photodetectors is based on the generation of photocarriers by the injection of an optical signal into a semiconductor/metal, where detected signals are obtained as a photocurrent according to the envelope of the injected optical signal to the detectors. Therefore, amplitude/intensitymodulated optical signals can be detected directly. However phase/frequency-modulated optical signals cannot. The speed of standard photodetectors is limited by the transit time of the photocarriers through the device or an RC time constant. Therefore, detection efficiency decreases as optical signal frequency increases, and signal detection modulated at high frequency ranges over millimeter-waves is rather difficult except for specifically tailored ultra-fast detectors like the unitraveling-carrier photodiode (UTC-PD) [1] .

Difference frequency generation (DFG) based on a second-order nonlinear optical effect is another candidate for the detection of a high-speed optical signal [2-4]. Using DFG, it is possible to shift the spectrum of a modulated optical signal from lightwave frequency ranges to micro/millimeter-wave frequency ranges directly. Therefore, not only amplitude/intensity-modulated optical signals, but also phase/frequency-modulated signals are applicable. In next generation optical fiber communication networks, several types of advanced vector modulation signals (frequency shift keying (FSK)/phase shift keying (PSK)/amplitude and phase shift keying (APSK)) are important for extremely high-bitrate data transfer. The DFG technique is applicable to the conversion of optical FSK/PSK/APSK signals, which are hard to detect directly using conventional photocurrent based devices. The conversion of optical orthogonal frequencydivision multiplexing (OFDM) signals to a microwave frequency range is also possible by using the DFG technique.

Several studies on signal generation/conversion based on DFG from a second-order nonlinear optical effect have been reported [2-4]. However, the conversion efficiency is low due to the difficulty of phase matching which comes from the differences in the velocities between the lightwaves and the micro-/millimeter waves. Furthermore, the Manley-Rowe relationship, which expresses the difference in the photon energy between lightwaves and micro-/millimeter waves [5], causes even lower output power.

In this paper, we present the detection of an optical signal modulated at a microwave frequency using DFG in periodically-poled $\mathrm{LiTaO}_{3}$ with a microwave resonator structure composed of a straight microwave waveguide. In order to obtain high conversion efficiency, a periodically poled structure of ferroelectric optical crystal is adopted for quasi-phase-matching (QPM) between the lightwave and the microwave. The microwave Fabry-Perot resonator is also utilized for the enhancement of the output signal 
by the resonance effect. This device has the following advantages: it has a simple structure, only the optical signal at the target frequency is converted, the other optical signals including an optical carrier can pass through the device without disturbance, peak detection frequency can be tailored by tuning the polarization reversal period and the resonance condition, and the signal conversion efficiency becomes greater at higher frequencies unlike conventional photodetectors.

In the following sections, the device structure, analysis, design, and experimental results of the proposed device are presented.

\section{Device Structure}

Figure 1 shows the structure of the DFG-based optical signal detection device we have proposed. $\mathrm{LiTaO}_{3}$ is adopted as a nonlinear optical material for DFG. $\mathrm{LiNbO}_{3}$ or other ferroelectric optical crystals with second-order optical nonlinearity are also applicable. The surfaces of the four sidewalls of the long rectangular $\mathrm{LiTaO}_{3}$ crystal are covered with a thin metal film in order to construct a microwave rectangular waveguide. Both ends of the rectangular $\mathrm{LiTaO}_{3}$ crystal are uncovered for light beam coupling and microwave output. A channel optical waveguide structure is also applicable for confining and guiding lightwaves along the crystal. The $\mathrm{LiTaO}_{3}$ crystal is periodically poled for QPM between the lightwave and the microwave. By DFG in the $\mathrm{LiTaO}_{3}$ crystal, a microwave signal is generated when the modulation frequency of the input optical signal coincides with the designed frequency determined by the poling period and the microwave resonance condition.

Periodic poling structure is designed for QPM between the modulated lightwave and the generated microwave. In DFG for optical signal detection, the frequency difference between the two lightwaves, which corresponds to the frequency of the generated microwave, is rather small compared with their individual frequencies. Therefore, the quasi-velocity-matching (QVM) scheme between the light group velocity and the microwave phase velocity [6] is useful for the design of the poling period for the QPM.

The structure of the microwave resonator is just a FabryPerot cavity. Since the refractive index value of $\mathrm{LiTaO}_{3}$ in the microwave frequency range is rather large $(\sim 6.5)$ compared with air, about half of the microwave signal generated through DFG in the $\mathrm{LiTaO}_{3}$ crystal is reflected at both ends of the microwave rectangular waveguide and propagates backwards and forwards in it. As a result, the Fabry-Perot cavity can be constructed without specific mirrors/reflectors in the microwave frequency ranges.

\section{Analysis and Design}

In the DFG shown in Figure 1, the coupling between the two lightwave modes, with angular frequencies $\omega_{1}$ and $\omega_{2}$, and the microwave mode of $\omega_{3}$ propagating to the $+y$ direction is described by using coupled-mode equations assuming the slowly varying amplitude approximation [5]

$$
\begin{aligned}
& j \frac{d A_{1}}{d y}=\kappa_{1} \Gamma A_{2} A_{3} \exp \left[-j\left(\beta_{2}+\beta_{3}-\beta_{1}\right) y\right], \\
& j \frac{d A_{2}}{d y}=\kappa_{2} \Gamma A_{1} A_{3}^{*} \exp \left[-j\left(\beta_{1}-\beta_{3}-\beta_{2}\right) y\right], \\
& j \frac{d A_{3}}{d y}=\kappa_{3} \Gamma A_{1} A_{2}^{*} \exp \left[-j\left(\beta_{1}-\beta_{2}-\beta_{3}\right) y\right],
\end{aligned}
$$

where $A_{1}, A_{2}$, and $A_{3}$ are the complex amplitudes of the electric field of each mode, $\kappa_{1}, \kappa_{2}$, and $\kappa_{3}$ are the coupling constants determined by the nonlinear coefficient of the crystal and the polarization of the three modes, $\Gamma$ is the parameter defined by the overlap of the field distribution of the three modes, and $\beta_{1}, \beta_{2}$, and $\beta_{3}$ are the phase constants of the three modes.

The key point of the device design is to utilize a single guided-mode structure for the output microwave signal at the designed frequency. If there are several microwave guided modes or radiation modes in the device at the designed frequency range, the nonlinear polarization induced by the second order nonlinear optical effect might be simultaneously coupled to several modes propagating with different phase constants. Then, the generated signal by DFG might be spread out over these modes, and it is difficult to obtain high conversion efficiency.

Here, we set $A_{3}$ as the mode in the microwave frequency range and introduce a microwave waveguide with a rectangular structure. Adopting the periodically poled structure, the sign of the three coupling constants $\left(\kappa_{1}, \kappa_{2}\right.$, and $\left.\kappa_{3}\right)$ is modulated along the propagation direction. Therefore, the phase-mismatching among the three modes can be compensated for and high-efficiency coupling can be obtained. The length, $L$, of each polarization-reversed/nonreversed region to realize efficient DFG for the microwave at angular frequency $\omega_{3}=\omega_{1}-\omega_{2}=2 \pi f_{m}$ is expressed by the following equation:

$$
\begin{aligned}
L & =\frac{\pi}{\left|\beta_{1}-\beta_{2}-\beta_{3}\right|} \\
& \approx \frac{\pi}{\left|(\partial \beta / \partial \omega)\left(\omega_{1}-\omega_{2}\right)-\beta_{3}\right|} \\
& =\frac{\pi}{\left|\left(n_{g} / c\right) \omega_{3}-\left(n_{m} / c\right) \omega_{3}\right|} \\
& =\frac{c}{2 f_{m}\left(n_{m}-n_{g}\right)},
\end{aligned}
$$

where $n_{g}$ is the group index of the lightwaves which is expressed by $n_{g}=c(\partial \beta / \partial \omega), n_{m}$ is the effective index of the microwave mode in the rectangular waveguide, and $c$ is the lightwave velocity in vacuum. This final transformed equation is equivalent with the QVM condition in travelingwave electro-optic modulators [6].

It is clear from (2) that there is an optimum detection frequency for the poling period. Therefore, the proposed detection device is a band-operating device. The frequency 


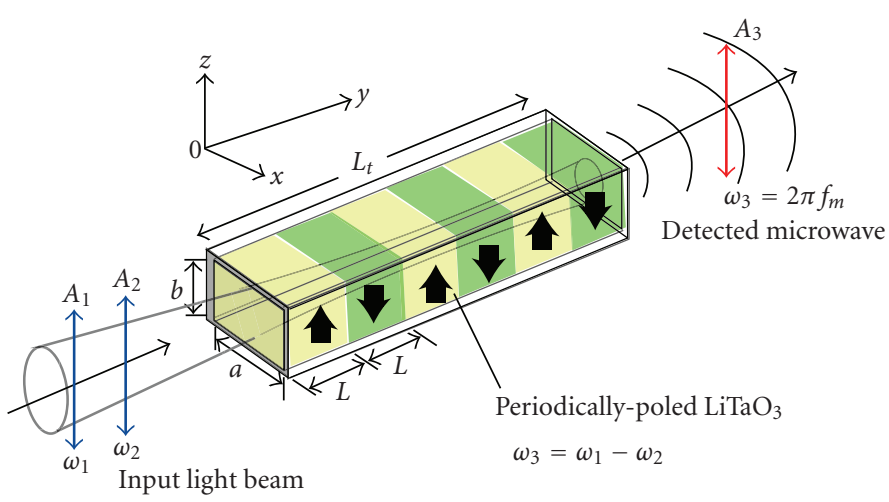

(a)

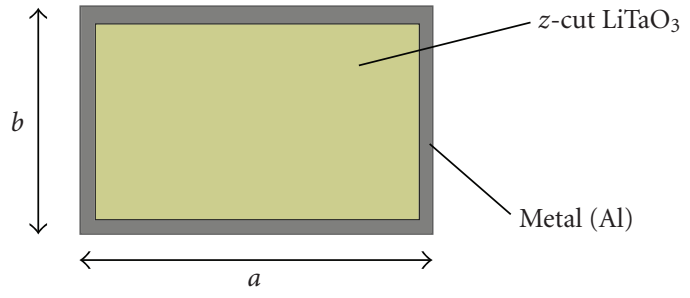

(b)

FIGURE 1: Basic structure of the optical signal detection device with periodically poled structure and microwave rectangular waveguide. The whole structure is (a), and its cross sectional view is (b).

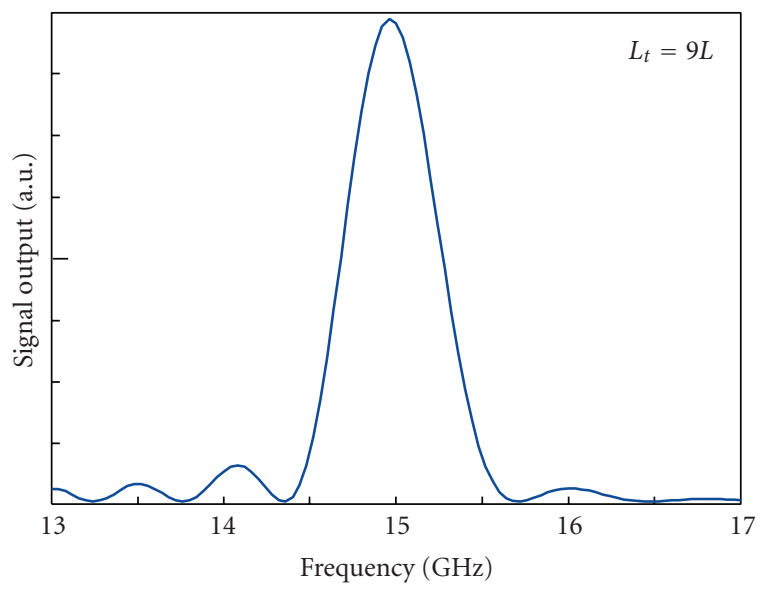

FIGURE 2: Calculated frequency dependence by the QPM.

response of the signal detection determined by the QPM can be calculated using the coupled-mode equation (1) taking into account the reversal of the sign of the coupling coefficient by polarization reversal. The calculated example is shown in Figure 2, where the total device length, $L_{t}$, was set as $L_{t}=9 L$. The QPM bandwidth is inversely proportional to the total device length $L_{t}$. The frequency response of the proposed device is also dependent on the resonance condition of the Fabry-Perot cavity structure. Therefore, the final frequency response is determined by the multiplication of the response by the QPM shown in Figure 2 and the response by the Fabry-Perot resonator.

In order to utilize the largest nonlinear coefficient of the $\mathrm{LiTaO}_{3}$ crystal, $d_{33}$, a $z$-cut $\mathrm{LiTaO}_{3}$ crystal substrate was adopted and the $z$-axis of the crystal was set parallel to the shorter side in the cross-section. Under this configuration, the polarization directions of the two lightwave modes that we set were the same and parallel to the $z$-axis. Therefore, the generated microwave by the DFG with the $d_{33}$ nonlinear coefficient was polarized along the $z$-axis. This means that the TE guided mode in the microwave rectangular waveguide can be obtained. The calculated dispersion relationship of the $\mathrm{TE}_{10}$ mode is shown in Figure 3. By setting the length of the shorter side of the crystal to an appropriate value, the microwave rectangular waveguide only supports a $\mathrm{TE}_{10}$ mode in the designed frequency range and a high-efficiency DFG signal generation can be expected.

\section{Experiments}

The prototype device was fabricated using a $z$-cut $\mathrm{LiTaO}_{3}$ substrate. First, a periodically poled structure was fabricated in a $0.4 \mathrm{~mm}$ thick crystal substrate by use of the pulse voltage applying method. The period of the poling, $2 L$, was set to $10.2 \mathrm{~mm}$, where the designed lightwave wavelength was $1550 \mathrm{~nm}$ (the group index of the extraordinary light is $n_{g}=2.17$ ) and the peak detection frequency was $15 \mathrm{GHz}$ using a microwave rectangular waveguide, with a crosssection of $2.0 \times 0.4 \mathrm{~mm}$ (the effective index of the $\mathrm{TE}_{10}$ mode is $n_{m}=4.13$ at $15 \mathrm{GHz}$ ). Next, the periodically poled crystal was cut with a diamond saw to $45.9 \mathrm{~mm}$ along the propagation direction and $2.0 \times 0.4 \mathrm{~mm}$ in crosssection, which was designed for the cutoff frequency of the microwave rectangular waveguide at $11.6 \mathrm{GHz}$ and for a single-mode structure with a frequency range from $11.6 \mathrm{GHz}$ to $23.1 \mathrm{GHz}$. After cutting, both ends of the crystal were polished. Finally, a $2 \mu \mathrm{m}$ thick Al film was deposited on the four sidewalls of the crystal by use of electron-beam vapor deposition.

The microwave resonance characteristics of the fabricated device were measured by use of a pair of microwave probe antennas and a network analyzer. The measured microwave transfer characteristic through the fabricated device is shown in Figure 4. The resonance peaks in a FabryPerot cavity were clearly observed. The measured separation of the adjoining resonance frequency, $\Delta f$, was $\Delta f \sim 1 \mathrm{GHz}$ around $15 \mathrm{GHz}$, which was in good agreement with the calculated value from the following equation:

$$
\Delta f=\frac{c}{2 L_{t} n_{m}}
$$

where $c$ is the lightwave velocity in vacuum, $L_{t}$ is the total length of the device, and $n_{m}$ is the effective index of 


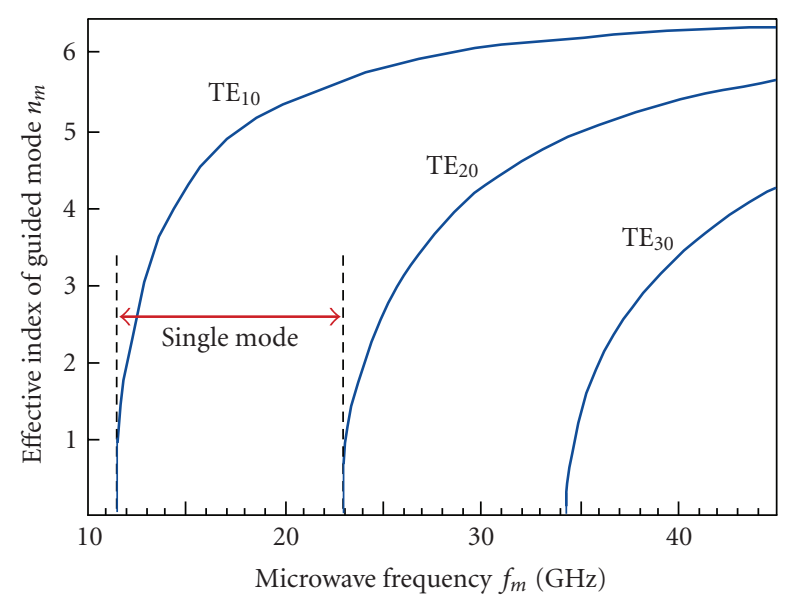

(a)

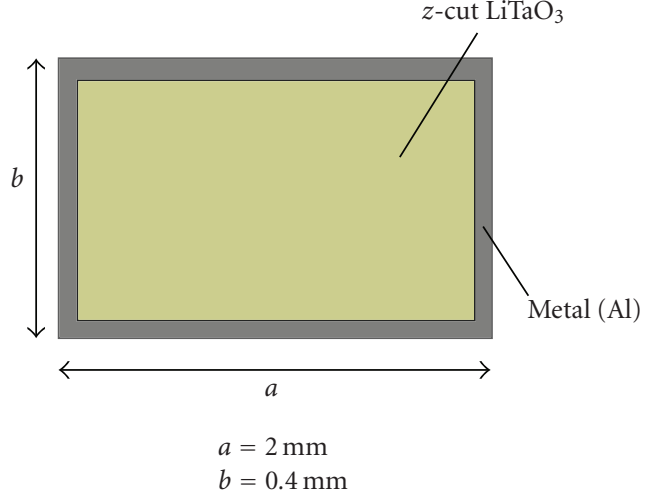

(b)

FIgURE 3: Calculated dispersion characteristics of the guided modes in the $\mathrm{LiTaO}_{3}$ microwave rectangular waveguide with $a=2$ mm and $b=0.4 \mathrm{~mm}$.

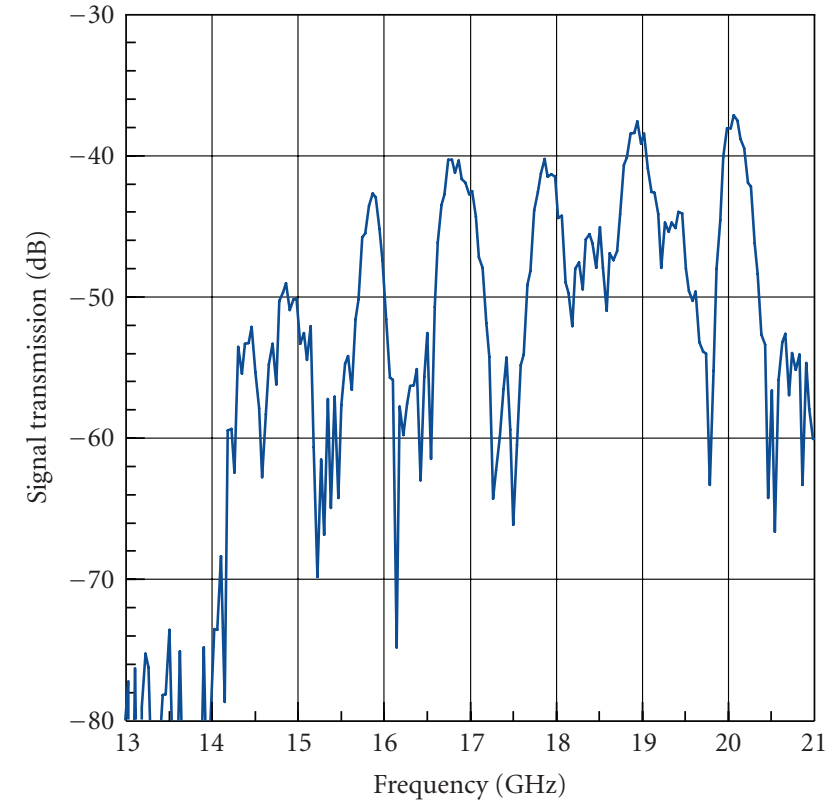

FIGURE 4: Measured microwave resonance characteristics of the fabricated device.

the microwave $\mathrm{TE}_{10}$ mode propagating in the rectangular waveguide, which was calculated as $\sim 4$ in the designed frequency range. Therefore, a single-mode microwave propagation characteristic in the designed frequency range was confirmed experimentally. The measured unloaded Q-factor around each resonance frequency was $\sim 50$. In Figure 4 , the transmission signal is rather small below $14 \mathrm{GHz}$, which was due to the cutoff characteristics of the microwave probe antennas used in the measurement.

The experimental setup for optical signal detection is shown in Figure 5. The light source was a $1550 \mathrm{~nm}$ CW DFB laser diode. The CW lightwave from the laser diode was deeply modulated by use of a high-speed optical inten- sity modulator with a driving modulation frequency from $13 \mathrm{GHz}$ to $18 \mathrm{GHz}$ and amplified by use of an Erbium-doped optical fiber amplifier. The intensity modulated lightwave with a power of $20 \mathrm{~mW}$ was focused on the end surface of the fabricated device using a lens of a $50 \mathrm{~mm}$ in focal length. The output microwave signal, which was emitted from the end of the DFG device, was measured using a microwave horn antenna and a microwave spectrum analyzer. An example of the detected signal spectrum is shown in Figure 6. A clear signal was observed at $15.1 \mathrm{GHz}$. The measured frequency response of the detected signal is shown in Figure 7. The peak detection frequency was $15.1 \mathrm{GHz}$, which was in good agreement with the expected characteristics calculated from the frequency response by the QPM (Figure 2) and the measured microwave resonance characteristics of the fabricated device (Figure 4). The detected signal power level $(\sim 0.1 \mathrm{pW})$ was comparable with the estimated value using the $d_{33}$ nonlinear coefficient of $\mathrm{LiTaO}_{3}$, the microwave frequency, the light wavelength, the device length and crosssection, the unloaded Q-factor of the Fabry-Perot resonator, and the coupling efficiency between the device and the horn antenna used in the measurement.

\section{Discussion and Conclusion}

In the experiment, the coupling between the DFG device and the microwave horn antenna was small $(<-10 \mathrm{~dB})$, since the radiated microwave from the device rapidly spread out from the end of the rectangular waveguide with a small crosssection of $2.0 \times 0.4 \mathrm{~mm}$, which is rather small compared with the wavelength of the emitted microwave in air of $20 \mathrm{~mm}$. By using an appropriate microwave circuit for increasing the coupling efficiency such as a microwave lens or a large aperture array of antennas, we expect to enhance the output power by $\sim 10 \mathrm{~dB}$. The application of an optical waveguide structure is also attractive for increasing the conversion efficiency with diffraction-less light propagation over a long interaction length. It should be noted that the output power 


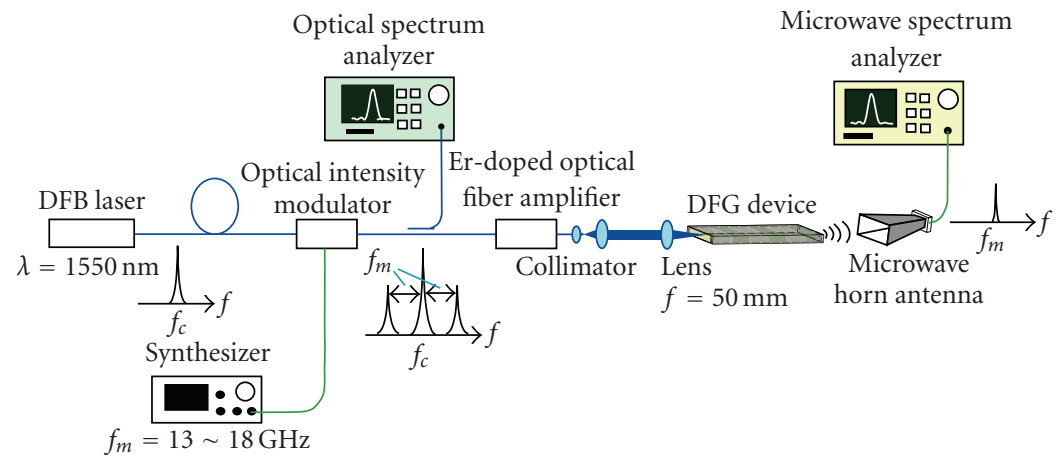

FIGURE 5: Experimental setup for the detection of an optical signal modulated at $\sim 15 \mathrm{GHz}$.

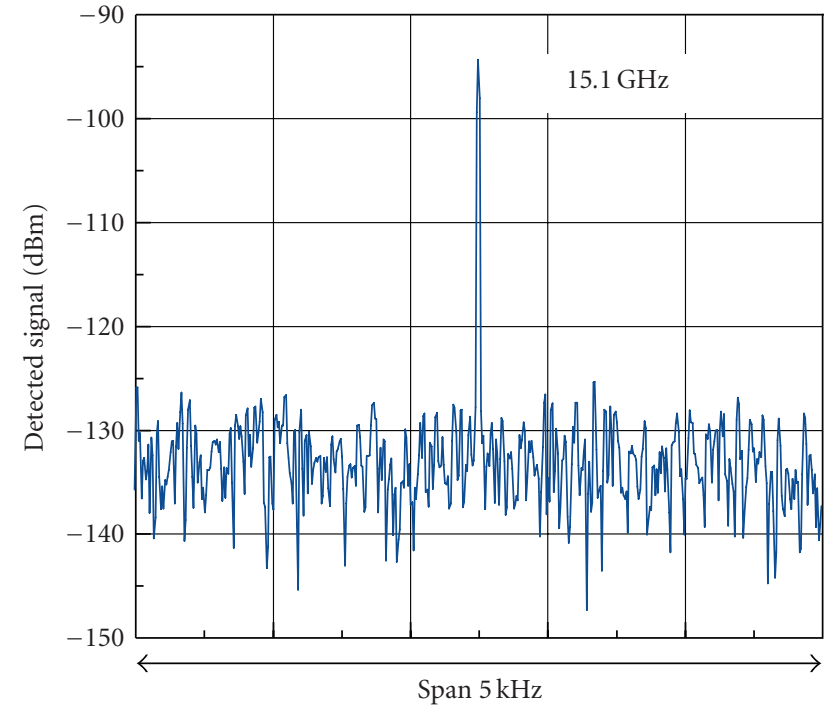

Figure 6: Measured spectrum of the detected signal. The modulation frequency was $15.1 \mathrm{GHz}$ and the input lightwave power was $20 \mathrm{~mW}$.

level of the detected signal is proportional to the square of the signal frequency. Therefore, as the signal frequency becomes higher, the larger conversion efficiency is obtainable. By increasing the output power with these techniques, it is expected that the detection of optical clock signals at a highrepetition frequency without disturbance from other data signals would be obtainable. This would be useful for the next generation optical communication networks.

An optical heterodyne scheme injecting another phaselocked laser beam to the DFG device is also applicable for converting the signal to other frequency ranges, which is useful for extracting subcarrier multiplexed signals in orthogonal frequency-division multiplexing (OFDM) schemes or radio-on-fiber (ROF) systems.

\section{Acknowledgments}

The authors thank Drs. Akira Enokihara and Hidehisa Shiomi for their valuable advice and Toshiki Iwai and Ngo Quang Hong for their help with the analysis and the

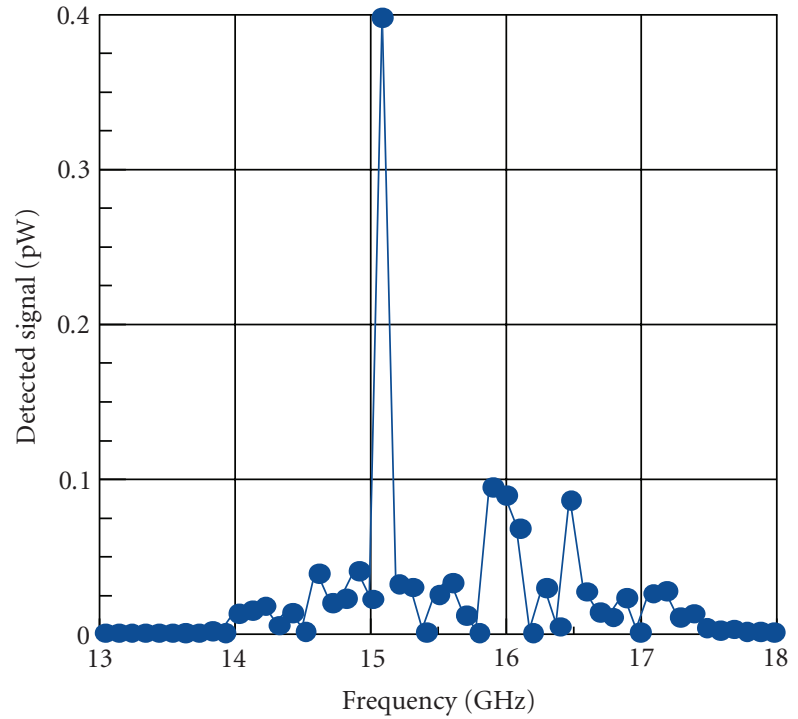

Figure 7: Measured frequency dependence of the detected signal with the input lightwave power of $20 \mathrm{~mW}$.

experiments. This work was supported in part by the Grants-in-Aid for Scientific Research from the Ministry of Education, Science, Sports and Culture, Japan.

\section{References}

[1] T. Ishibashi, S. Kodama, N. Shimizu, and T. Furuta, "Highspeed response of uni-traveling-carrier photodiodes," Japanese Journal of Applied Physics, vol. 36, no. 10, pp. 6263-6268, 1997.

[2] K. Kawase, M. Sato, T. Taniuchi, and H. Ito, "Coherent tunable THz-wave generation from $\mathrm{LiNbO}_{3}$ with monolithic grating coupler," Applied Physics Letters, vol. 68, no. 18, pp. 2483-2485, 1996.

[3] T. Hori, K.-H. Park, T. Kawanishi, and M. Izutsu, "Generation of CW millimeter wave signals in a lithium niobate nonlinear optical waveguide using modulated optical input," Japanese Journal of Applied Physics, vol. 39, no. 7A, pp. L667-L669, 2000.

[4] K. Suizu, T. Shibuya, S. Nagano, et al., "Pulsed high peak power millimeter wave generation via difference frequency generation using periodically poled lithium niobate," Japanese Journal of Applied Physics, vol. 46, part 2, no. 36-40, pp. L982-L984, 2007. 
[5] A. Yariv, Quantum Electronics, John Wiley \& Sons, New York, NY, USA, 3rd edition, 1989.

[6] H. Murata, A. Morimoto, T. Kobayashi, and S. Yamamoto, "Optical pulse generation by electrooptic-modulation method and its application to integrated ultrashort pulse generators," IEEE Journal on Selected Topics in Quantum Electronics, vol. 6, no. 6, pp. 1325-1331, 2000. 

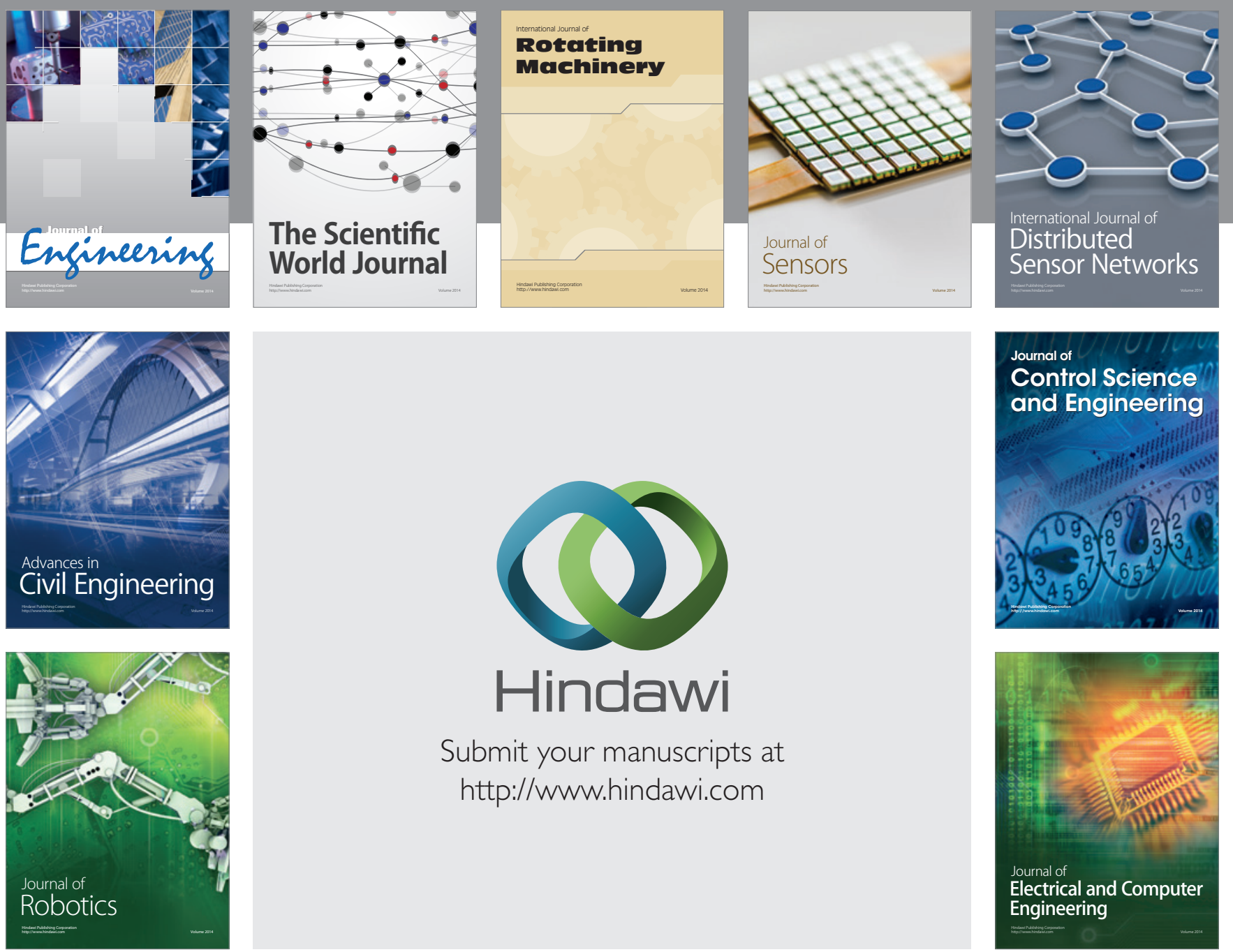

Submit your manuscripts at

http://www.hindawi.com
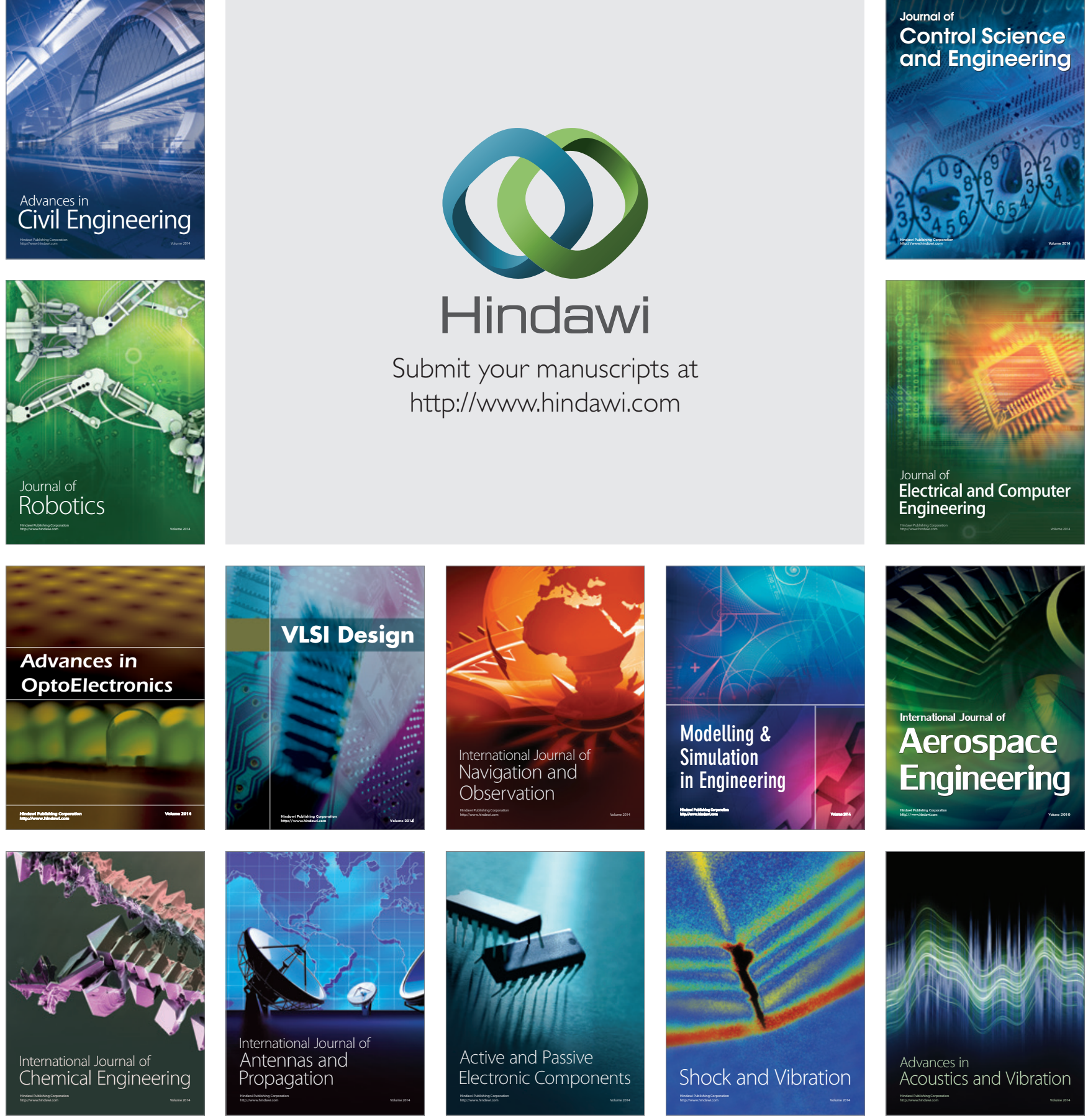